\title{
Psychophysical Evaluation of Change in Friction on an Ultrasonically-Actuated Touchscreen
}

\author{
Muhammad Khurram Saleem, Cetin Yilmaz, and Cagatay Basdogan
}

\begin{abstract}
To render tactile cues on a touchscreen by friction modulation, it is important to understand how human perceive a change in friction. In this study, we investigate the relations between perceived change in friction on an ultrasonically actuated touchscreen and parameters involved in contact between finger and its surface. We first estimate the perceptual thresholds to detect rising and falling friction while finger is sliding on the touch surface. Then, we conduct intensity scaling experiments and investigate the effect of finger sliding velocity, normal force, and rise/fall time of vibration amplitude (transition time) on the perceived intensity of change in friction. In order to better understand the role of contact mechanics, we also look into the correlations between the perceived intensities of subjects and several parameters involved in contact. The results of our experiments show that the contrast and rate of change in tangential force were best correlated with the perceived intensity. The subjects perceived rising friction more strongly than falling friction, particularly at higher tangential force contrast. We argue that this is due to hysteresis and viscoelastic behavior of fingertip under tangential loading. The results also showed that transition time and normal force have significant effect on our tactile perception.
\end{abstract}

Index Terms-Tactile perception, ultrasonic vibrations, surface haptics

\section{INTRODUCTION}

$\mathbf{T}$ OUCHSCREENS are an integral part of mobile phones, tablets, laptops, kiosks, and information display panels. They enable us to interact with digital content via finger gestures. Although they can be used to detect the positions of fingers, and track their trajectory to recognize the applied gesture, the tactile feedback displayed to the user through commercial touchscreens today is limited to simple vibrations, which is typically used to inform the user about the incoming calls and provide confirmation when typing a text or pressing a digital button. However, the user still has to pay constant attention to the visual content displayed on the screen to perform even simple tasks such as dialing a number on a mobile phone. Moreover, although the size of touchscreens used in mobile devices has increased drastically during the last few years, allowing a larger area for touch interactions, the digital content displayed through those devices is still predominantly visual, which increases in amount every day and overloads our brain. Instead, if some of the content is displayed through the haptic channel, it may alleviate our cognitive load. Furthermore, integrating vision and haptics

M.K. Saleem and C. Basdogan are with the Department of Mechanical Engineering, Koc University, Istanbul, Turkey, 34450. Email: ksaleem13@ku.edu.tr, cbasdogan@ku.edu.tr

C. Yilmaz is with the Department of Mechanical Engineering, Bogazici University, Istanbul, Turkey, 34342. Email:cetin.yilmaz@ boun.edu.tr to display information in a multi-modal form can enhance task performance and user perception. For example, in design of user interfaces, the intensity of frictional tactile feedback displayed to a user while dragging a folder on a touchscreen can be adjusted based on the amount of data contained in the folder (e.g. more friction for the folders containing more data). Tactile feedback may not only help the user to drag the folder to the target location with more precision but also appreciate its size intuitively during dragging without explicitly inspecting its content. Displaying tactile feedback through a touchscreen may also improve user engagement and experience. For example, displaying not only the color of a fabric but also its texture through tactile channel may improve user experience during online shopping.

As these motivating examples show, the techniques to provide sophisticated tactile sensations on touchscreens are highly desirable. Currently, there are two prominent techniques, namely electrostatic and ultrasonic actuation, for displaying tactile feedback through the surface of a touchscreen. In both techniques, the aim is to modulate the frictional forces between the surface and fingertip, though their working principles are different. In electrostatic actuation, a voltage is applied to the conductive layer of a touchscreen to generate an attractive force between its surface and a finger sliding on it, which leads to an increase in frictional forces applied to the finger opposite to the direction of movement [1]. On the other hand, when a touch surface is actuated at an ultrasonic resonance frequency, friction is reduced between the surface and finger. Two different mechanisms have been suggested to explain the cause of friction reduction in ultrasonic tactile displays; Watanabe et al. [2] proposed that the friction reduction is due to the formation of a squeezed film of air between finger and the surface. Alternatively, it has been suggested that, when a surface vibrates at an ultrasonic frequency, an intermittent mechanical contact develops between finger and the surface such that the finger bounces on the surface while sliding [3][5]. A recent study conducted by Wiertlewski et al. [6] using a stroboscope revealed that both mechanisms indeed contribute to the friction reduction. Hence, it appears that the fingertip bounces on a cushion of squeezed film of air. Ultrasonic actuation can provide a higher variation in friction as compared to the electrostatic actuation, while the rendering bandwidth of ultrasonic devices is limited due to its resonating nature [7]. However, it is possible to implement electrostatic and ultrasonic actuation techniques together on the same surface to achieve even larger variation in friction coefficient [8].

A touch surface can be made slippery/sticky using ultrasonic actuation technique, simply by turning the vibrations ON/OFF. 
In order to render shapes and surface features such as textures, friction has to be modulated, based on the finger position or velocity [9]. Hence, in order to modulate friction on a touch surface properly, it is important to understand our tactile perception of change in friction and the contact mechanics behind it. Although, the earlier studies have investigated the contact mechanics of friction [5], [10]-[17] and our perceptual ability to discriminate two different surfaces based on friction [18]-[20], the number of studies investigating the intermittent contact mechanics during a change in friction on a touch surface is very limited [21]-[24]. Furthermore, the factors affecting our tactile perception of this change have not been investigated in depth yet [22]-[26].

\section{A. Literature Review}

1) Friction on Real Surfaces: The earlier studies in tribology literature show that friction between skin and a smooth glass surface is mainly governed by interfacial interaction (adhesion), while the contribution due to viscoelastic deformations is reported to be low. In case of a smooth surface, increasing the normal force increases tangential force, while the coefficient of friction decreases [10]-[12], [15], [16]. Andre et al. [11] reported that the effect of normal force on friction coefficient is valid below $3.5 \mathrm{~N}$ only. Similarly, the contribution in friction due to viscoelastic deformations decreases rapidly with an increase in normal force, as reported by Derler et al. [12]. The friction between a smooth surface and fingertip also varies with moisture, lipids/water ratio, occlusion time, finger velocity, and fingertip roughness [11], [13], [14], [16], [21].

Friction plays an important role in human perception of surface features. The ability of humans to discriminate smooth surfaces based on friction was studied by Smith and Scott [18]. They found that the subjects could identify microscopically smooth surfaces with a great accuracy using active touch. Although, the friction coefficients of the surfaces used in their study were very low, yet a higher correlation found between the estimated friction and the measured one. Smith et al. [27] investigated the role of friction and tangential force in tactile perception of roughness using linear gratings. They found a positive correlation between friction and roughness perception, however rate of change in tangential force correlated better with roughness estimates. Friction is also important in the discrimination of wet and dry surfaces. Chen et al. [28] found that the subjects perceived a surface as dry if the friction and compliance of the surface were low. The above studies suggest that friction is indeed very important in tactile perception. Nonetheless, a recent study showed that two surfaces can be perceived different during tactile exploration, even though they have the same frictional properties, due to the difference in molecular interactions between finger and the surfaces [19].

2) Friction on Ultrasonically Actuated Surfaces: In an experimental study investigating friction reduction on an ultrasonically actuated plate [17], the authors observed that coefficient of friction reduces as vibration amplitude is increased, as expectedly, but there was a limit in which no further improvements could be achieved. Moreover, the change in friction was small for higher normal forces and at values higher than $1 \mathrm{~N}$, there was in fact no perceivable change. Their results also showed that the finger velocity affects the friction coefficient at lower normal loads. On the other hand, Sednaoui et al. [5] reported that reduction in friction coefficient using ultrasonic vibrations depends on exploration velocity (tested at 20,40 , and $80 \mathrm{~mm} / \mathrm{s}$ ) but independent of normal force (tested at $0.2,0.5$, and $0.75 \mathrm{~N}$ ). In [21], Cornuault et al. have investigated the friction contrast on real and ultrasonically actuated surfaces. They found that the resulting contrast in friction depends on moisture and fingertip roughness, while no correlation was found between fingertip curvature and the spatial period of the ridges in finger skin.

There are very few studies in literature on friction perception during sliding on ultrasonically actuated surfaces. Samur et al. [20] have conducted discrimination experiments to evaluate the minimum detectable difference in friction using the tactile pattern display (TPad). The subjects were presented with two stimuli in sequential order and asked to identify the stimulus with higher friction. An average JND of $18 \%$ was reported for the friction difference. This study shows the ability of humans to discriminate two surfaces based on friction, but how humans perceive a change in friction cannot be ascertained. In this regard, Messaoud et al. [22] have evaluated the subjects' performance in detecting a change in friction. Their results showed that the detection rate improves at lower inherent friction between finger and surface, as well as lower finger velocity of $5 \mathrm{~mm} / \mathrm{s}$ compared to $20 \mathrm{~mm} / \mathrm{s}$. They also found that the detection rate is best correlated to friction contrast and a contrast greater than 0.19 is always detectable. It is shown in [23] that rate of change in tangential force is best correlated with the detection rate of friction change. Gueorguiev et al. [25] investigated the tactile perception of ultrasonic square signals displayed by friction modulation and observed that subjects could differentiate between two ultrasonic signals if the duration and transition time are extended by 2.4 and $2.1 \mathrm{~ms}$, respectively. In another experiment, they found that if the duration between two pulses was shorter than $50 \mathrm{~ms}$, subjects perceived them as a single pulse. In [29], authors have evaluated the threshold to detect two friction reductions of 100 ms duration, rendered $3 \mathrm{~mm}$ apart, using three different ultrasonically actuated surfaces made of aluminum, polypropylene and polyurethane. The rise time of vibration amplitude was controlled at $1.5 \mathrm{~ms}$. The surfaces were passively scanned under the finger at $20 \mathrm{~mm} / \mathrm{s}$, while normal force was maintained at $0.7 \mathrm{~N}$. They conducted threshold experiments and measured the vibration thresholds at $75 \% \mathrm{JND}$ as $0.17,0.23,0.27 \mu \mathrm{m}$ for aluminum, polypropylene and polyurethane, respectively. Furthermore, the detection rate was found to correlate well with the ratio of reduction in tangential force to pre-stimulation tangential force.

Apart from the studies on sliding-finger, it has been shown that a click effect, as in key press, can be created by reducing the friction as the user presses on an ultrasonically actuated touch surface [23], [26], [30], [31]. Tashiro et al. [30] have rendered the feeling of buckling and restitution of mechanical buttons using Langevin-type ultrasonic transducer. They argued that the perception of button press and release are due 
to momentary slippage of the finger over the surface, which occurs when the friction of the surface is reduced by ultrasonic actuation. Unlike [30], Monnoyer et al. [31] argued that the feeling of click occurs due to a sudden release of stress at the fingertip, which is accumulated when the friction of surface is high. Recently, it has been shown that there is an optimal value of finger impedance that leads to a stronger perception of haptic click effect [26]. Moreover, the rate of change in normal force has been reported to play an important role in perception of click effect [23].

\section{B. Research Objectives}

Earlier studies on friction have mainly focused on measuring the contact forces acting on fingertip moving on a frictional surface, estimating the coefficient of friction from the measured forces, and investigating their relation with our tactile perception. Although, there are also studies investigating our perceptual ability to discriminate two different surfaces based on friction, the number of studies investigating the change in friction on the same surface is very limited. The objective of this study is to investigate the relation between our tactile perception of friction change and the underlying contact mechanics during sliding. We have conducted psychophysical experiments to evaluate human tactile perception of friction change for rising friction $(R F)$ and falling friction $(F F)$, owing to the huge contrast in perception between them reported for pressing-finger [31]. First, we measure the detection thresholds under the experimental conditions of $R F$ and $F F$ for sliding-finger and pressing-finger (no intentional sliding). The second part of the study focuses on intensity scaling of friction change for sliding-finger. We performed intensity scaling experiments at different vibration amplitudes $\left(V_{a m p}\right)$ above the threshold value estimated in the first experiment, finger velocities $\left(f_{v}\right)$, transition times $\left(t_{r}^{a m p}\right)$, and mean normal forces $\left(F_{n}^{\text {mean }}\right)$. Here, the transition time $\left(t_{r}^{a m p}\right)$ is defined as the duration in which the vibration amplitude is linearly increased from zero to the desired value $\left(V_{a m p}\right)$ or vice-versa. Hence, transition time affects the slope of the friction change (e.g. longer transition time indicates slower change in friction).

\section{PSYCHOPHYSICAL EXPERIMENTS}

\section{A. Variable Friction Tactile Display}

The tactile display used in our study is a $100 \times 60 \mathrm{~mm}^{2}$ glass surface of $1.4 \mathrm{~mm}$ thickness. We actuate it at $26.9 \mathrm{kHz}$ using two piezoelectric patches (7BB-35, Murata Manufacturing) and a high voltage amplifier (PZD700A M/S, Trek). At the selected vibration mode, the distance between the nodal lines is approximately $11 \mathrm{~mm}$, creating an area of $11 \times 60 \mathrm{~mm}^{2}$ between two nodal lines for tactile exploration as shown in Fig. 1. A force sensor (Nano17 Titanium, ATI Industrial Automation) capable of measuring up to $8 \mathrm{~N}$ with a resolution of $1.5 \mathrm{mN}$ is used to record the normal and tangential forces acting on finger during the experiments. To acquire the vibration amplitude during contact interactions, a small piezoelectric patch (FT10.5T, Kepo Electronic) is used as a sensor. We have used an analog $R M S$-circuit to record the vibration amplitude at a lower sampling rate. The piezoelectric sensor is calibrated by

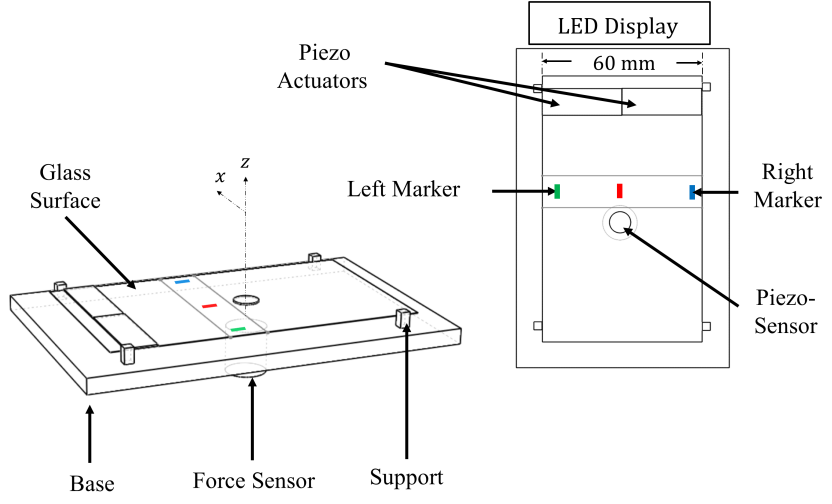

Fig. 1. Illustration of variable friction tactile display used in the study.

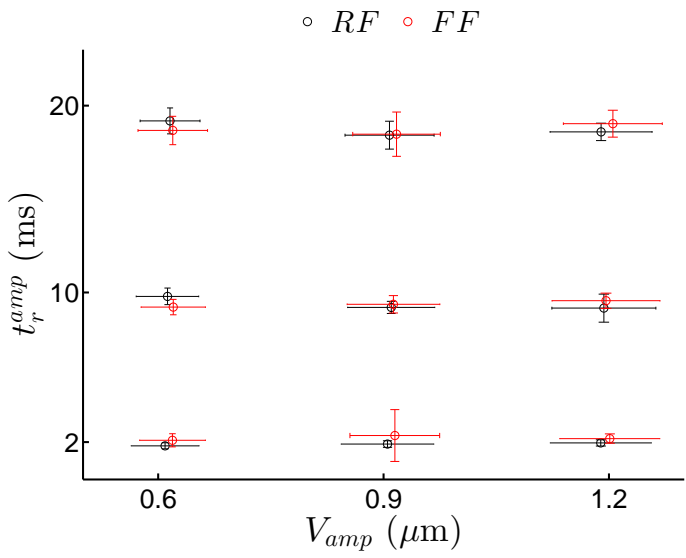

Fig. 2. Means and standard deviations for $V_{a m p}$ and $t_{r}^{a m p}$ recorded during the experiments. Tick values on horizontal and vertical axes are the desired values for $V_{a m p}$ and $t_{r}^{a m p}$, respectively

a Laser Doppler Vibrometer (LDV) (OFV-551, Polytec). To record the force and vibration amplitude simultaneously, we have used two data acquisition cards (PCI-6034E, National Instruments) and (PCIe-6321, National Instruments) running at $5 \mathrm{k}$ samples/seconds.

To create different tactile effects on the touch surface using ultrasonic actuation technique, vibration amplitude $\left(V_{a m p}\right)$ and transition time $\left(t_{r}^{a m p}\right)$ are used as the main control parameters. Finger damping during contact may cause fluctuations in $V_{a m p}$ and an active control might be required [32], [33]. However, in our experiments, the recorded vibration amplitude showed reasonable precision and accuracy without any active control, as depicted in Fig. 2.

\section{B. Threshold Estimation Experiments}

The first part of the study focuses on finding the smallest change in friction that humans can detect. We measured the perceptual thresholds for a) sliding-finger and b) finger pressed on the surface (no intentional sliding).

1) Experimental Design: The threshold estimation experiments were conducted using the method of constant stimuli [34]. There were two experimental conditions; rising friction $(R F)$ and falling friction $(F F)$. We rendered each condition at 


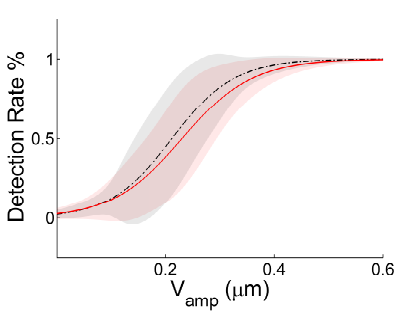

(a)

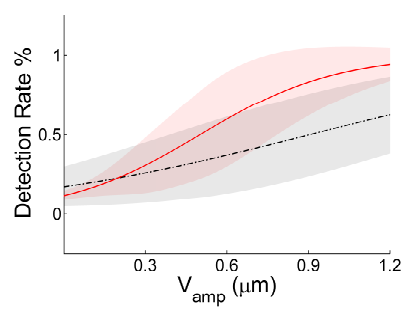

(b)
Fig. 3. Psychometric responses for threshold experiments. (Black-dotted: $R F$, red-solid: $F F$ ). Shaded region depicts standard deviation in detection rate. (a) Sliding-finger and (b) finger pressed on the surface (no sliding).

TABLE I

VIBRATION AMPLITUDE $(\mu \mathrm{m})$ AT MEAN THRESHOLD LEVELS

\begin{tabular}{l|l|cc} 
& $\begin{array}{l}\text { Friction } \\
\text { Change }\end{array}$ & $50 \%$ & $75 \%$ \\
\hline Sliding & $R F$ & $0.21 \pm 0.02$ & $0.27 \pm 0.02$ \\
finger & $F F$ & $0.22 \pm 0.02$ & $0.30 \pm 0.02$ \\
\hline $\begin{array}{l}\text { Pressing } \\
\text { Finger }\end{array}$ & $F F$ & $0.50 \pm 0.04$ & $0.78 \pm 0.06$ \\
\hline
\end{tabular}

different vibration amplitudes $\left(V_{a m p}\right)$ by altering the actuation voltage applied to the piezoelectric patches. The transition time $\left(t_{r}^{a m p}\right)$ was chosen as $2 \mathrm{~ms}$ in these experiments. The touch surface was actuated at 10 different vibration amplitudes (corresponding to 10 different voltage levels) varying from 0 to $0.6 \mu \mathrm{m}$ in sliding-finger experiment, and from 0 to $1.2 \mu \mathrm{m}$ in pressing-finger experiment. Each stimulus was repeated 10 times, hence each subject completed 200 trials ( 2 conditions $\times 10$ different voltage levels $\times 10$ repetitions). The trials were displayed in a random order, while the same order was displayed to each subject.

2) Procedure: In sliding-finger experiment, the subjects explored the surface from left to right only. The subjects were instructed to synchronize their finger velocity with a visual cursor moving with a velocity of $(50 \mathrm{~mm} / \mathrm{s})$. We changed the friction as the subjects' finger crossed the mid-point of the exploration region, which was detected by an IR-frame (see "RED" mark in Fig. 1). The subjects were asked to respond to the following question by saying YES/NO; "Did you feel any tactile effect?"

In pressing-finger experiment, the subjects were asked to press their finger on the touch surface marked in "RED". Therefore, the spatial location where we changed the friction was same for both threshold estimation experiments. We changed the friction when the normal force $\left(F_{n}\right)$ applied by finger exceeded $0.3 \mathrm{~N}$. A LED display turned $\mathrm{ON}$ to indicate that there was a change in tactile stimuli and then subjects were asked to respond to the following question by saying YES/NO; "Did you feel a click?"

Ten subjects participated in the threshold estimation experiment ( 7 males, 3 females, average age of $27 \pm 3$ ). The subjects read and signed the consent form before the experiments. The form was approved by Ethical Committee for Human Participants of Koc University.

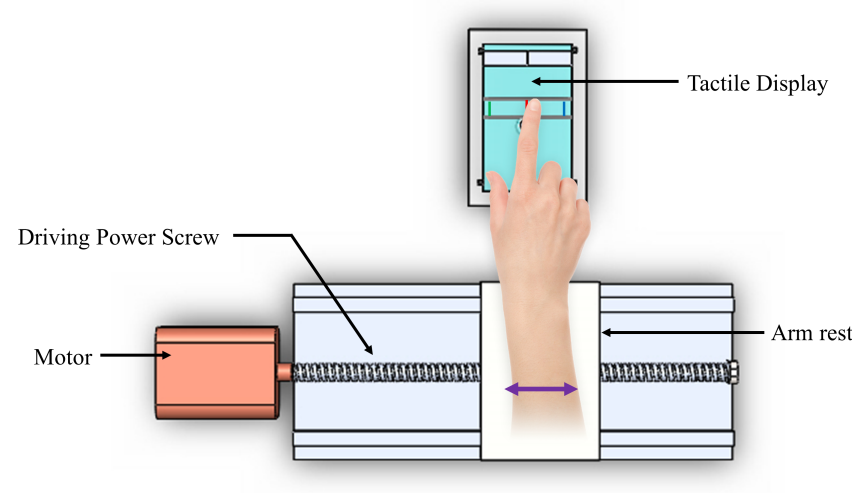

Fig. 4. Illustration of experimental setup used in intensity scaling experiments.

3) Results: We fitted a logistic function to the mean response of each subject for $R F$ and $F F$, separately. Then, an average curve for all subjects for $R F$ and $F F$ was constructed from the individual curves and threshold values were reported based on those average curves. The results of sliding-finger experiment showed that subjects' perception of $R F$ and $F F$ was quite similar (Fig. 3(a)), and followed a typical psychometric response $\left(R^{2}=0.98\right)$. The paired $\mathrm{t}$ test unveiled that the difference between $R F$ and $F F$ was not significant $(p=0.08)$. The results of the pressing-finger experiment are shown in Fig. 3(b). We found that the response for $F F$ followed a typical psychometric behavior $\left(R^{2}=0.99\right)$, whereas $R F$ was not perceived as a click and curve fit was poor $\left(R^{2}=0.5\right)$. The paired t-test showed that the difference between $R F$ and $F F$ was significant $(p<0.001)$. Our results are in line with the recent literature [31]. The vibration amplitudes $\left(V_{a m p}\right)$ for the mean detection rates of 50 and 75 $\%$ are given in Table I (For pressing-finger experiment, data for $F F$ condition was reported only). For further details on our threshold experiments, the readers are referred to [23].

The threshold experiments showed that $R F$ and $F F$ were perceived equally when finger was sliding, whereas a pressingfinger could only detect $F F$. Moreover, the perceptual threshold for $F F$ in pressing-finger experiment was twice the value measured for the sliding-finger experiment. The threshold experiment provided us valuable information about the tactile limits. However, the ability of humans to estimate the intensity of friction change cannot be ascertained from a threshold experiment. In the next section, we explore how subjects rate the intensity of friction change under different experimental conditions.

\section{Intensity Scaling Experiments}

In the second part of the study, we assessed the subjects' perception of change in friction intensity during sliding. We have chosen our vibration amplitudes $\left(V_{a m p}\right)$ significantly above the detection levels estimated for the sliding-finger in the first experiment. We altered finger velocity $\left(f_{v}\right)$, transition time $\left(t_{r}^{a m p}\right)$, and the mean normal force applied to the surface $\left(F_{n}^{\text {mean }}\right)$ in a controlled manner to investigate if they cause 

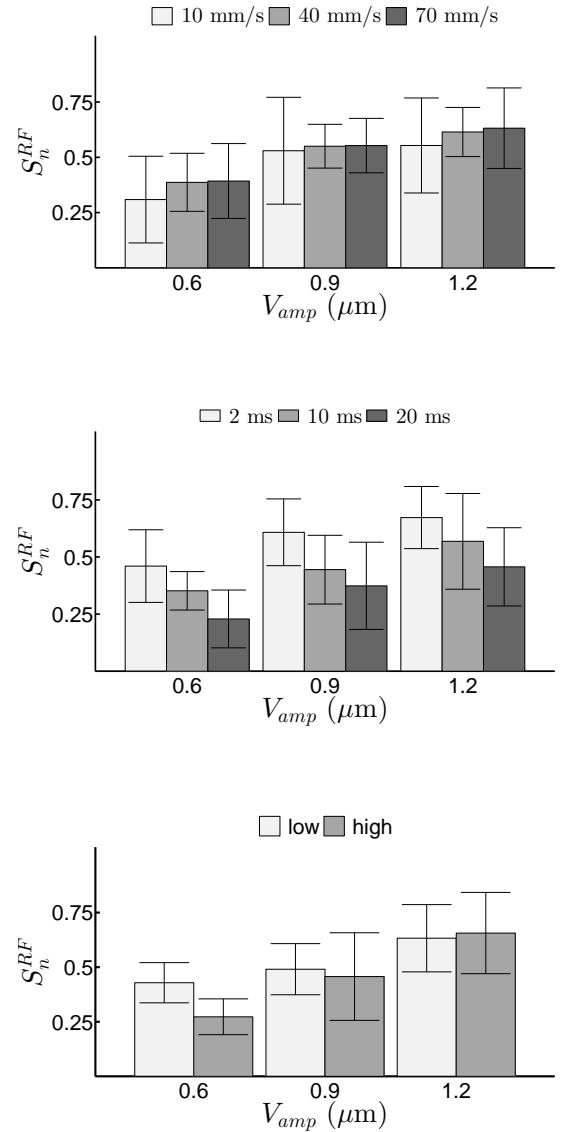

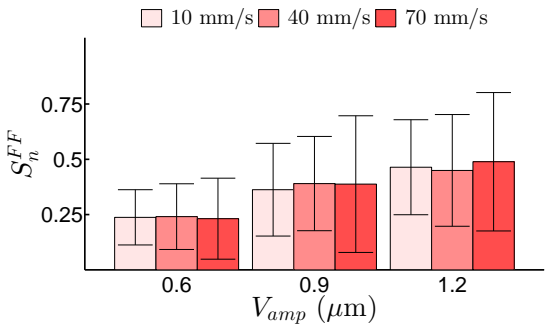

(a)

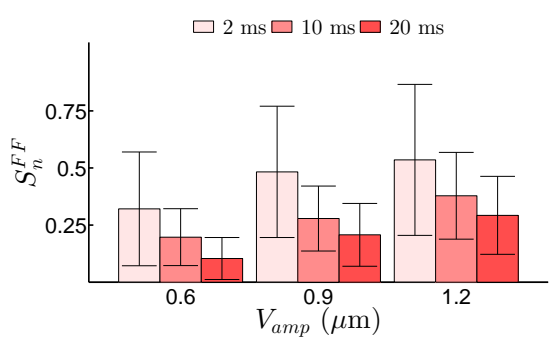

(b)
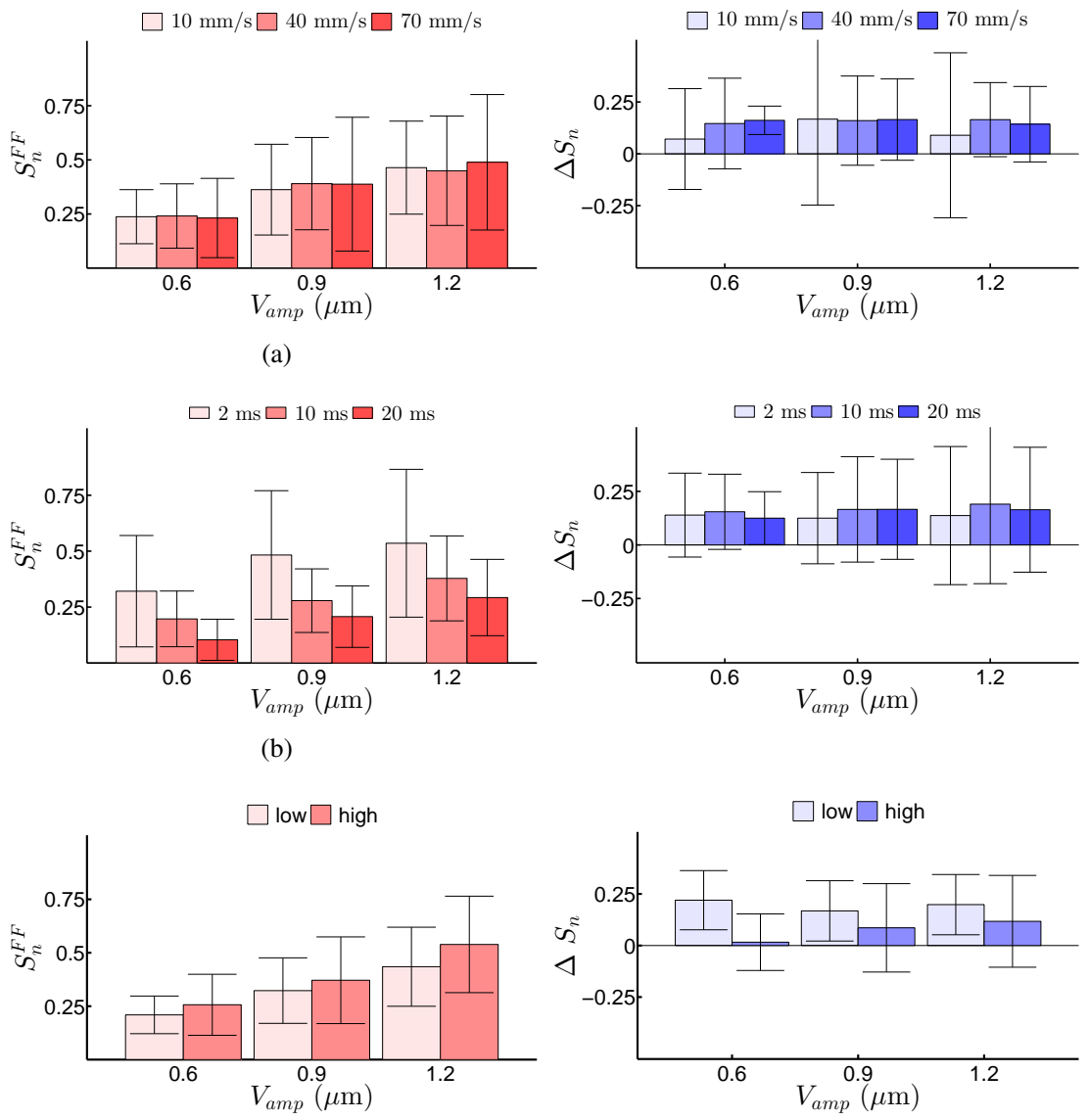

(c)

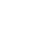


itself. The trials were randomized such that each rendering condition displayed equally for the movements from left to right and right to left. We used a computer fan to continuously blow air over the touch surface to minimize the undesirable effects of moisture. Moreover, the subjects were instructed to lift their finger up at the end of each trial to release accumulated stresses. A white noise was played through active noise canceling headphones to prevent perceptual bias due to any auditory noise. In $E x p-1$ and $E x p-2$, the subjects were instructed to press the surface gently, like touching the surface of a mobile phone or a tablet. There was one minute break after every 36 trials, during which the finger of subject was cleaned with alcohol. In $E x p-3$, we displayed a reference value (i.e. desired value) for the normal force to the subjects through a GUI $(0.15 \mathrm{~N}$ for "low" force and $0.45 \mathrm{~N}$ for "high" force). The reference force was changed after every 12 trials, preceding a break of one minute (note that we did not change the reference force after each trial because our preliminary experiments showed that the subjects could not adapt well to this procedure).

Eleven subjects participated in the experiments ( 9 males, 2 females, average age of 27 \pm 4 ). The subjects read and signed the consent form before the experiments. The form was approved by Ethical Committee for Human Participants of Koc University.

3) Results: First, we normalized the scores $\left(S_{n}\right)$ of each subject to vary between zero and one. We observed inconsistent responses for a few subjects, perhaps due to misunderstanding of the task or lack of concentration during the task. Therefore, we measured within-subject variability by computing coefficient of variation $(C V)$ and then calculated interquartile range $(I Q R)$ in $C V$ s for all subjects. The data of subjects with $C V$ s more than $1.5 \times I Q R$ above the upper quartile were rejected (as a result, the data of $2 / 11$ subjects in $\operatorname{Exp}-1$ and $\operatorname{Exp}-3$, while 1/11 subject in $\operatorname{Exp}-2$ was rejected). The results showed that subjects scored $R F$ higher than $F F$ as shown in Fig. 5. Nonetheless, this trend was not same among the subjects. We conducted paired ttests and categorized the subjects in three groups accordingly; a) those who scored $R F$ higher than $F F$ (with $p<0.05$ ) are called RF-biased group $\left(G_{R F}\right)$, b) those who scored $F F$ higher than $R F$ are called FF-biased group $\left(G_{F F}\right)$, and c) those who perceived $R F$ and $F F$ equally $(p \geq 0.05)$ are called neutral group $\left(G_{N}\right)$. The number of subjects in each group are listed in Table II.

The results of intensity scaling experiments are summarized below:

- In all experiments, increasing $V_{a m p}$ increased $S_{n}$ as expected.

- In all experiments, average values of $S_{n}$ for $R F$ was higher than that of $F F(p<0.01)$. Therefore, the difference between scores $\left(\Delta S_{n}=S_{n}^{R F}-S_{n}^{F F}\right)$ was positive.

- The results of $\operatorname{Exp}-1$ showed that there was no significant effect of $f_{v}$ on $S_{n}$.

- In Exp - 2, increasing $t_{r}^{a m p}$ decreased $S_{n}$. All paired ttests between $S_{n}$ values for $t_{r}^{a m p}=2,10,20 \mathrm{~ms}$ returned $p<0.001$.
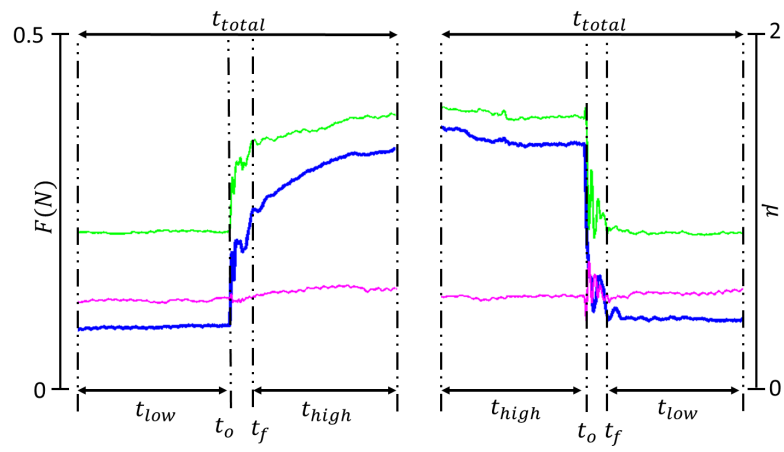

Fig. 6. An exemplar data window used for the computation of force metrics for $R F$ (left) and $F F$ (right). Blue, magenta and green colored lines represent $F_{t}, F_{n}$ and $\mu$, respectively.

- In $E x p-3$, paired t-test showed that $S_{n}$ values of subjects were higher under high normal force than those of the low normal force for $F F(p<0.05)$.

- Overall, $\Delta S_{n}$ was not influenced significantly by $f_{v}$ and $t_{r}^{a m p}$ but its absolute value decreased as $F_{n}^{\text {mean }}$ was increased. (See plots of $\Delta S_{n}$ in Fig. 5).

4) Analysis of Forces: Intensity scaling experiments showed that $R F$ and $F F$ were perceived differently by the subjects. To better understand the reasoning behind this, we analyzed the contact forces recorded during the experiments. Before the analysis, the force data was filtered using a low-pass filter with a cut-off frequency of $600 \mathrm{~Hz}$. The cut-off frequency was chosen to cover the sensitivity range of human finger [37]. For the analysis, we considered a window of data only, which was centered around the transition time and corresponded to the data collected for $70 \%$ of the total sliding time. We further sub-divided this window in time domain as elaborated in Fig. 6 to develop various force metrics (Table III). Calculating the transition time of force $\left(t_{r}^{\text {force }}=t_{f}-t_{o}\right.$ ) was challenging due to the variation in contact forces, even at constant stimulation level. To tackle with this problem, $t_{f}$ is defined as the time when the derivative of tangential force $\left(d F_{t} / d t\right)$ decays below $80 \%$ of its peak value after $t_{o}$, where $t_{o}$ is defined as the time when the friction change was rendered while the subjects' finger crossed "RED" marker. The values computed for $t_{r}^{\text {force }}$ are shown in Table IV. The results showed that, $t_{r}^{\text {force }}$ was significantly higher than $t_{r}^{a m p}$ for both $R F$ and $F F$, and $t_{r}^{f o r c e}$ in $R F$ was in general higher than that of $F F$. Moreover, $t_{r}^{\text {force }}$ at low scan speed of $10 \mathrm{~mm} / \mathrm{s}$ was significantly higher as compared to the typical scan speeds of $f_{v}=40$ and $70 \mathrm{~mm} / \mathrm{s}$. Meyer et al. [7] have also reported a higher force transition time in $R F$ for a lower velocity of $20 \mathrm{~mm} / \mathrm{s}$ and argued that it is due to slow discharge of squeeze film. Fig. 9(a) shows that the subjects were successful in attaining and maintaining the desired "low" and "high" normal forces in Exp -3 . We investigated the correlation between $S_{n}$ and normalized force metrics using Spearman correlation $(\rho)$ and the results were checked for the statistical significance level of $p<0.05$.

Following are the main results gathered from the force metrics and their correlation with $S_{n}$ : 


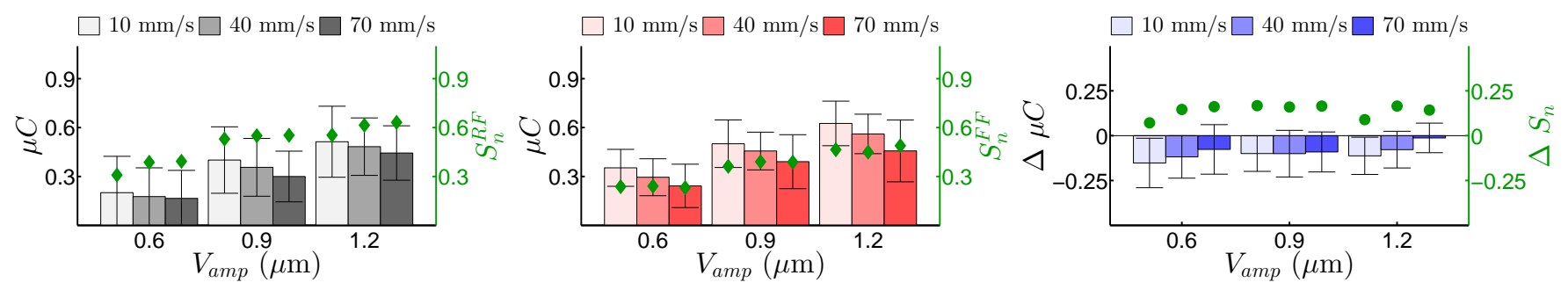

(a)

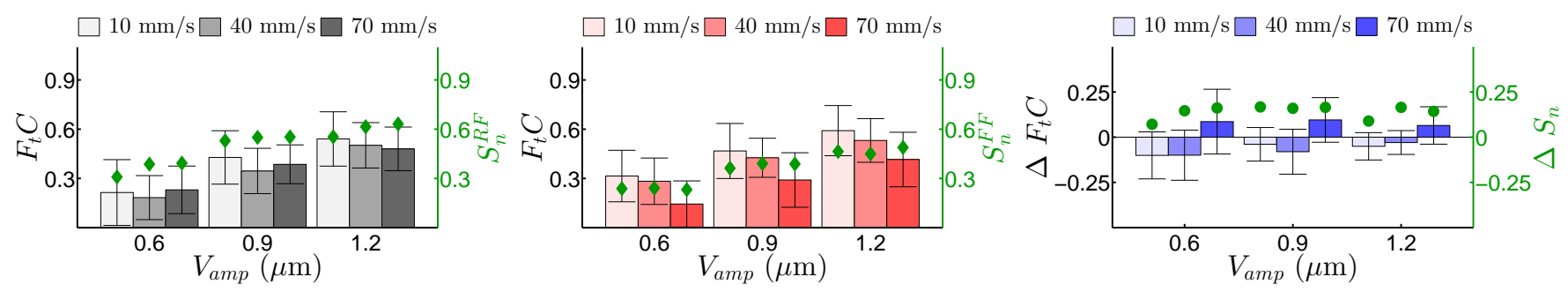

(b)
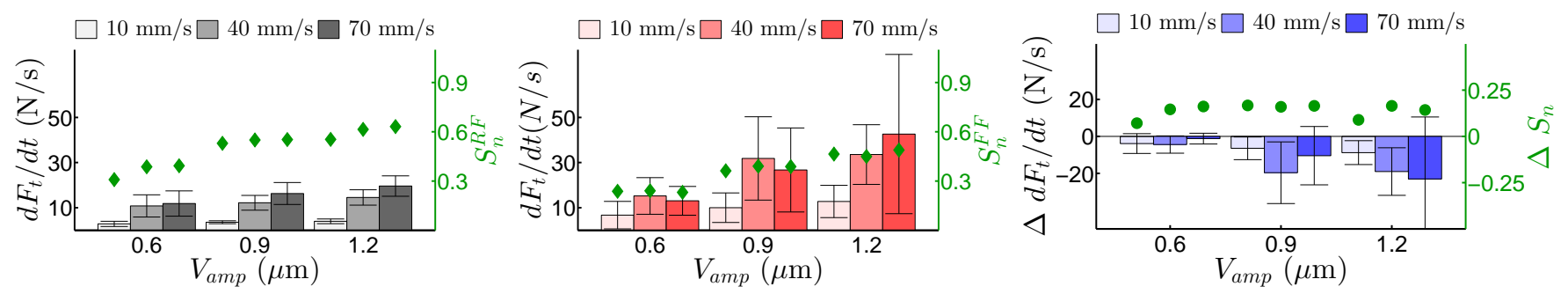

(c)

Fig. 7. Means and standard deviations of various force metrics for $E x p-1$. The first, second, and third column report the results for $R F, F F$, and difference between $R F$ and $F F$, respectively. Green-colored diamonds and circles represent mean values of $S_{n}$ and $\Delta S_{n}$, respectively (Refer to the values on the right axis in each plot).

TABLE III

Force Metrics, Their Symbols AND Descriptions

\begin{tabular}{|c|c|c|}
\hline Force Metric & Symbol & Description \\
\hline $\begin{array}{l}\text { Instantaneous: } \\
\text { Tangential Force } \\
\text { Normal Force } \\
\text { Friction Coefficient }\end{array}$ & $\begin{array}{c}F_{t} \\
F_{n} \\
\mu\end{array}$ & $\begin{array}{c}\text { Force in } \\
\text { sliding direction, } \\
\text { and normal direction. } \\
\mu=F_{t} / F_{n}\end{array}$ \\
\hline $\begin{array}{l}\text { Mean: } \\
\text { Tangential Force } \\
\text { Normal Force } \\
\text { Friction Coefficient }\end{array}$ & $\begin{array}{l}F_{t}^{\text {low }}, F_{t}^{\text {high }}, F_{t}^{\text {mean }} \\
F_{n}^{\text {low }}, F_{n}^{\text {high }}, F_{n}^{\text {mean }} \\
\mu^{\text {low }}, \mu^{\text {high }}, \mu^{\text {mean }}\end{array}$ & $\begin{array}{c}\text { Mean values at } \\
t_{\text {low }}, t_{\text {high }}, t_{\text {total }}\end{array}$ \\
\hline $\begin{array}{l}\text { Difference: } \\
\text { Tangential Force } \\
\text { Normal Force } \\
\text { Friction Coefficient }\end{array}$ & $\begin{array}{c}\Delta F_{t} \\
\Delta F_{n} \\
\Delta \mu\end{array}$ & $\begin{array}{c}F_{t}^{\text {high }}-F_{t}^{\text {low }} \\
F_{n}^{\text {high }}-F_{n}^{\text {low }} \\
\mu^{\text {high }}-\mu^{\text {low }}\end{array}$ \\
\hline $\begin{array}{l}\text { Contrast: } \\
\text { Tangential Force } \\
\text { Normal Force } \\
\text { Friction Coefficient }\end{array}$ & $\begin{array}{c}F_{t} C \\
F_{n} C \\
\mu C\end{array}$ & $\begin{array}{l}1-F_{t}^{\text {low }} / F_{t}^{\text {high }} \\
1-F_{n}^{\text {low }} / F_{n}^{\text {high }} \\
1-\mu^{\text {low }} / \mu^{\text {high }}\end{array}$ \\
\hline $\begin{array}{l}\text { Transition Time: } \\
\text { Tangential Force }\end{array}$ & $t_{r}^{\text {force }}$ & $t_{f}-t_{o}$ \\
\hline $\begin{array}{l}\text { Rate of change: } \\
\text { Tangential Force } \\
\text { Normal Force } \\
\text { Friction Coefficient }\end{array}$ & $\begin{array}{l}d F_{t} / d t \\
d F_{n} / d t \\
d \mu / d t\end{array}$ & $\begin{array}{l}\text { RMS values } \\
\text { for time interval } \\
\text { of } t_{r}^{\text {force }}\end{array}$ \\
\hline
\end{tabular}

TABLE IV

Transition Time of TANGENTIAL ForCE DURING THE EXPERIMENTS AT VARIOUS CONDITIONS

\begin{tabular}{|c|c|c|c|c|c|}
\hline & \multirow[t]{2}{*}{$\begin{array}{c}f_{v} \\
(\mathrm{~mm} / \mathrm{s})\end{array}$} & \multirow[t]{2}{*}{$\begin{array}{l}t_{r}^{a m p} \\
(\mathrm{~ms})\end{array}$} & \multirow[t]{2}{*}{$\begin{array}{c}F_{n}^{\text {mean }} \\
(\mathrm{N})\end{array}$} & \multicolumn{2}{|c|}{$\begin{array}{c}t_{r}^{\text {force }} \\
(\mathrm{ms})\end{array}$} \\
\hline & & & & $R F$ & $F F$ \\
\hline \multirow{3}{*}{$\operatorname{Exp}-1$} & 10 & 2 & - & $23 \pm 11$ & $22 \pm 14$ \\
\hline & 40 & 2 & - & $10 \pm 1$ & $6.9 \pm 2$ \\
\hline & 70 & 2 & - & $10 \pm 1$ & $6.9 \pm 2$ \\
\hline \multirow{3}{*}{$\operatorname{Exp}-2$} & 40 & 2 & - & $11 \pm 1$ & $7.2 \pm 3$ \\
\hline & 40 & 10 & - & $18 \pm 3$ & $18 \pm 8$ \\
\hline & 40 & 20 & - & $37 \pm 7$ & $29 \pm 8$ \\
\hline \multirow{2}{*}{$E x p-3$} & 40 & 2 & $<0.15$ & $10 \pm 2$ & $6 \pm 2$ \\
\hline & 40 & 2 & $>0.45$ & $10 \pm 2$ & $6.5 \pm 2$ \\
\hline
\end{tabular}

- It can be seen from Figs. 7, 8, and 10 that $S_{n}$ was correlated with $F_{t} C$ and $d F_{t} / d t$. Results of the correlation analysis (see TableV) also supported this observation.

- The force analysis of Exp-2 showed that $F_{t} C$ did not change much with $t_{r}^{a m p}$. Therefore, despite longer $t_{r}^{\text {force }}$, the contact forces reached a steady-state value within 40 $\mathrm{mm}$ of travel distance as shown in Fig. 8(a) and 8(b).

- In $E x p-3$, as $F_{n}^{\text {mean }}$ was increased, $F_{t} C$ reduced, while $d F_{t} / d t$ and $S_{n}$ increased (see Fig. 10). 

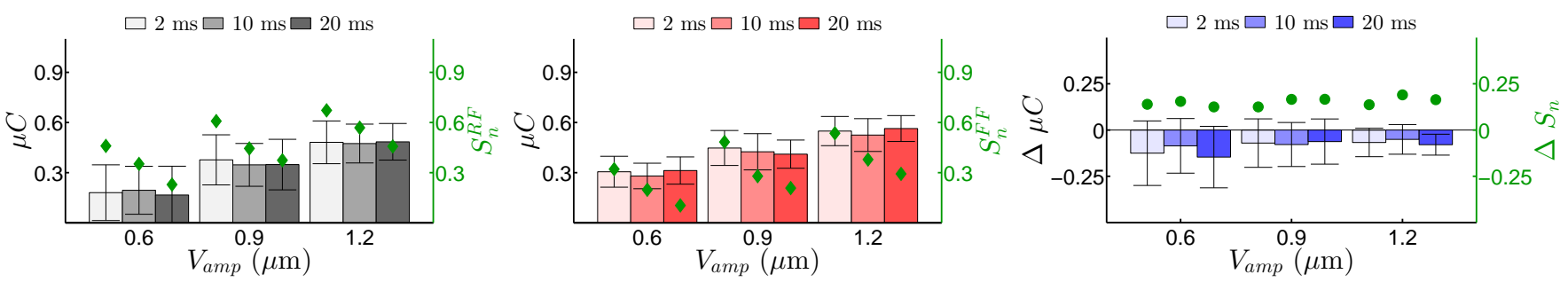

(a)
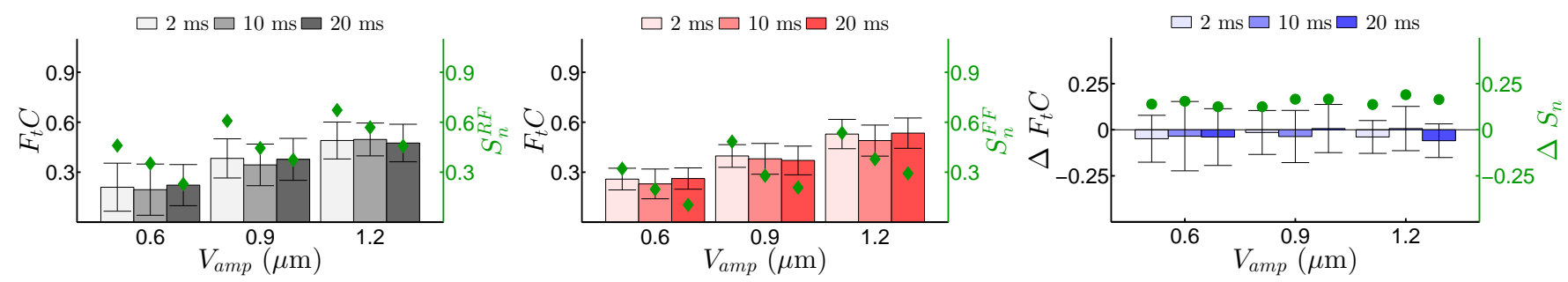

(b)
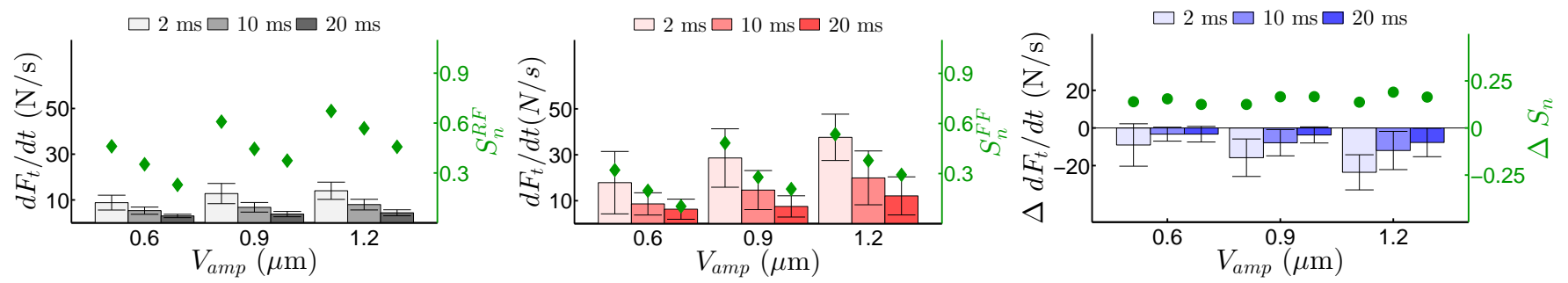

(c)

Fig. 8. Means and standard deviations of various force metrics for $E x p-2$. The first, second, and third column report the results for $R F, F F$, and difference between $R F$ and $F F$, respectively. Green-colored diamonds and circles represent mean values of $S_{n}$ and $\Delta S_{n}$, respectively (Refer to the values on the right axis in each plot).

TABLE V

Force Metrics Showing Highest Correlation with $S_{n}$

\begin{tabular}{c|ll|ll|ll} 
& $\mid$ Exp-1 & \multicolumn{2}{|c|}{ Exp -2} & Exp -3 \\
\cline { 2 - 7 } & Metric & $\rho$ & Metric & $\rho$ & Metric & $\rho$ \\
\hline \multirow{3}{*}{$R F$} & $F_{t} C$ & 0.59 & $d F_{t} / d t$ & 0.75 & $F_{t} C$ & 0.65 \\
& $\mu C$ & 0.56 & $t_{r}^{\text {force }}$ & -0.63 & $d F_{t} / d t$ & 0.63 \\
& $\Delta F_{t}$ & 0.54 & $F_{t} C$ & 0.62 & $\mu C$ & 0.63 \\
\hline \multirow{3}{*}{$F F$} & $F_{t} C$ & 0.67 & $d F_{t} / d t$ & 0.81 & $d F_{t} / d t$ & 0.59 \\
& $\mu C$ & 0.48 & $t_{r}^{\text {force }}$ & -0.79 & $t_{r}^{\text {force }}$ & -0.56 \\
& $d F_{t} / d t$ & 0.47 & - & - & $F_{t} C$ & 0.4 \\
\hline
\end{tabular}

5) Modeling: The results of all three experiments showed that $d F_{t} / d t$ and $F_{t} C$ were the most important metrics in intensity scaling of friction change. In order to find their relative contributions, we fitted a linear model using Matlab function (fitlm) to the normalized scores of subjects in the form of $S_{n}=c_{1}+c_{2} \times F_{t} C+c_{3} \times d F_{t} / d t$ using the normalized values of $F_{t} C$ and $d F_{t} / d t$ (see Table VI). We found that the contribution of $d F_{t} / d t$ was more prominent in Exp -2 and Exp -3 , while $F_{t} C$ had a stronger effect in Exp-1.

\section{DISCUSSION}

The analysis of contact forces showed that contrast in tangential force $\left(F_{t} C\right)$ and rate of change in tangential force $\left(d F_{t} / d t\right)$ were best correlated with the perceived intensity of friction change $\left(S_{n}\right)$. In fact, $S_{n}$ can be estimated quite accurately by a linear function of $F_{t} C$ and $d F_{t} / d t$, which suggests that there may be multiple sensory mechanisms involved in the perception of friction change. $F_{t} C$ causes a difference in magnitude of skin stretch while $d F_{t} / d t$ is more related to the vibrotaction. We argue that those are the two main mechanisms contributing to our tactile perception of change in friction. However, it is difficult to perform controlled experiments to assess their individual contribution to our perception since they are not easily separable from each other, as argued by [38]. In the upcoming paragraphs, we discuss the results of each experiment one by one and attempt to justify our argument.

In $E x p-1$, the finger velocity $\left(f_{v}\right)$ did not affect $S_{n}$. The analysis of forces showed that, $\mu C$ and $F_{t} C$ slightly decreased, while $d F_{t} / d t$ increased with increasing $f_{v}$ (see Fig. 7). Therefore, the combined effect of $F_{t} C$ and $d F_{t} / d t$ resulted in no significant change in $S_{n}$ as $f_{v}$ was increased. On the other hand, Messaoud et al. [22] have noted a significantly higher $\mu C$ at a lower velocity of $5 \mathrm{~mm} / \mathrm{s}$ as compared to $20 \mathrm{~mm} / \mathrm{s}$. Likewise, they observed higher detection rates for friction change at $5 \mathrm{~mm} / \mathrm{s}$. The difference in $\mu C$ reported in their study and ours could be due to a PVC film used in [22], while we conducted our experiments on a glass surface. Moreover, $\mu C$ is known to be affected by amount of moisture 


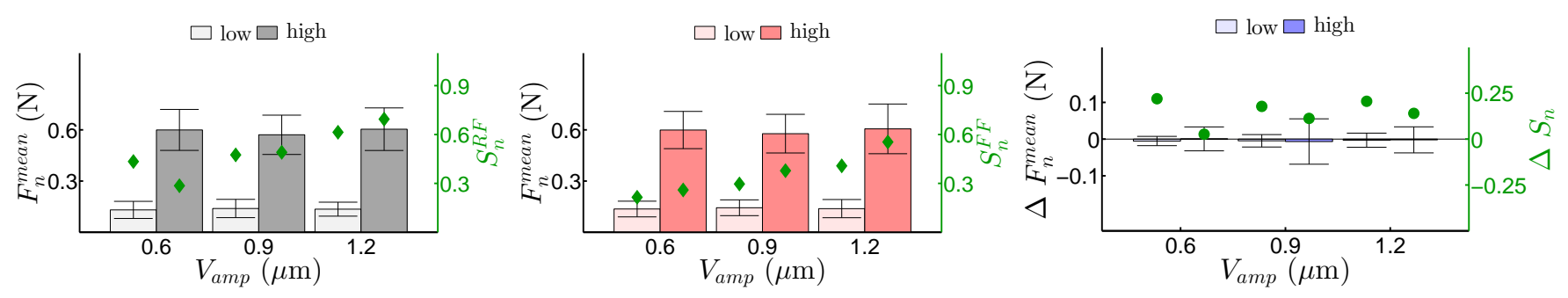

(a)
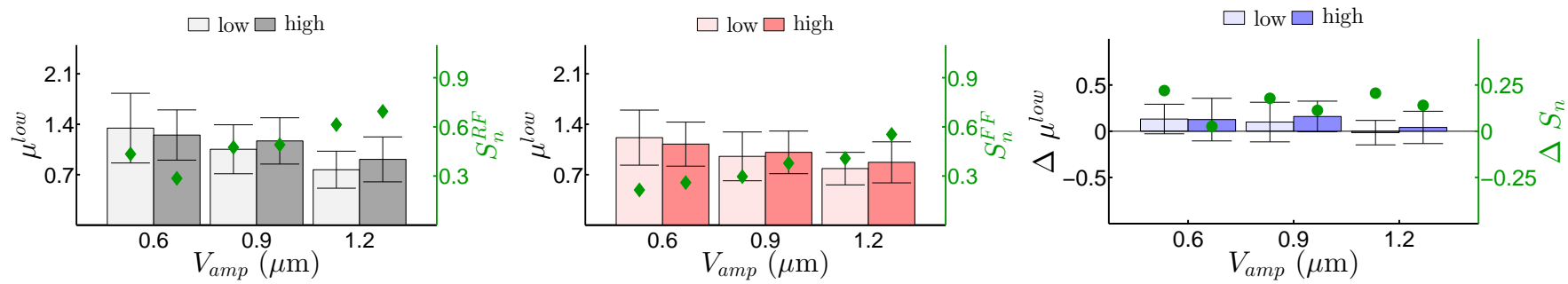

(b)
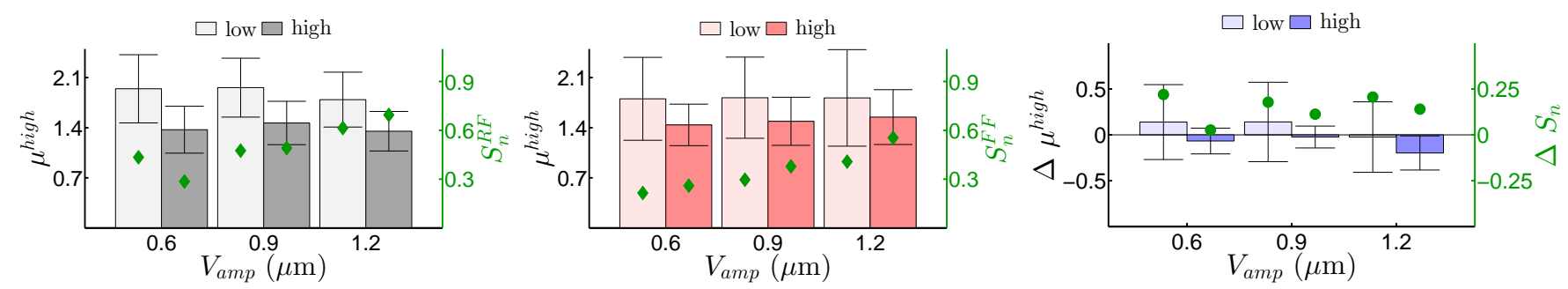

(c)

Fig. 9. Means and standard deviations of various force metrics for $E x p-3$. The first, second, and third column report the results for $R F, F F$, and difference between $R F$ and $F F$, respectively. Green-colored diamonds and circles represent mean values of $S_{n}$ and $\Delta S_{n}$, respectively (Refer to the values on the right axis in each plot).

TABLE VI

COEFFICIENTS OF LINEAR FIT FOR INTENSITY SCALING

\begin{tabular}{c|cccc|cccc} 
& \multicolumn{4}{|c}{$R F$} & \multicolumn{4}{c}{$F F$} \\
\cline { 2 - 9 } & $c_{1}$ & $c_{2}$ & $c_{3}$ & $R^{2}$ & $c_{1}$ & $c_{2}$ & $c_{3}$ & $R^{2}$ \\
\hline$E x p-1$ & -0.09 & 0.86 & 0.25 & $\begin{array}{l}0.94 \\
(p<0.001)\end{array}$ & -0.018 & 0.47 & 0.26 & $\begin{array}{l}0.89 \\
(p<0.001)\end{array}$ \\
\hline$E x p-2$ & 0.04 & 0.4 & 0.5 & $\begin{array}{l}0.97 \\
(p<0.001)\end{array}$ & 0.02 & 0.2 & 0.6 & $\begin{array}{l}0.93 \\
(p<0.001)\end{array}$ \\
\hline$E x p-3$ & -0.01 & 0.43 & 0.65 & $\begin{array}{l}0.89 \\
(p<0.05)\end{array}$ & -0.09 & 0.4 & 0.62 & $\begin{array}{l}0.96 \\
(p<0.001)\end{array}$ \\
\hline
\end{tabular}

and hydrolipid film between fingertip and touch surface [13], [21], which were not controlled in either studies.

In Exp - 2, $S_{n}$ decreased when friction was changed smoothly by making $t_{r}^{a m p}$ longer. In other words, when the rate of loading/unloading was higher (shorter transition time), the effect was stronger. We believe that $S_{n}$ decreased at higher $t_{r}^{a m p}$ because the change in tangential force no longer followed a sharp transition and could not strongly activate mechanoreceptors as discussed in [39] for electrovibration. A similar perceptual trend was reported for the magnitude estimation of skin stretch applied to human palm in [40]. On the other hand, Shull et al. [41] and Pare et al. [42] reported that rate of loading did not affect the magnitude estimates of torsional stretch applied to forearm, and force applied to fingertip, respectively (it is important to note that the loading rates of $0.1,0.15$, and $0.3 \mathrm{~N} / \mathrm{s}$ used in [42] were very low).

In $E x p-3, F_{t} C$ decreased significantly at high normal force $\left(F_{n}^{\text {mean }}\right)$, while $S_{n}$ did not change much. Derler et al. [12] reported that the contribution of viscoelastic deformations on the friction decreases as the normal force increases. Similarly, results of Exp -3 showed that increasing $F_{n}^{\text {mean }}$ decreased friction coefficient $\left(\mu^{\text {high }}\right.$ ) (see Fig. 9(c)). Interestingly, $\mu^{\text {low }}$ did not change much with $F_{n}^{\text {mean }}$ as shown in Fig. 9(b). For this reason, we observed a sharper drop in $\mu C$ at high $F_{n}^{\text {mean }}$. A similar perceptual effect for normal force was observed in a psychophysical experiment conducted to estimate the magnitude of slip on fingertip [43]. The authors argued that the fingertip gets stiffer at higher gripping force (as reported in 

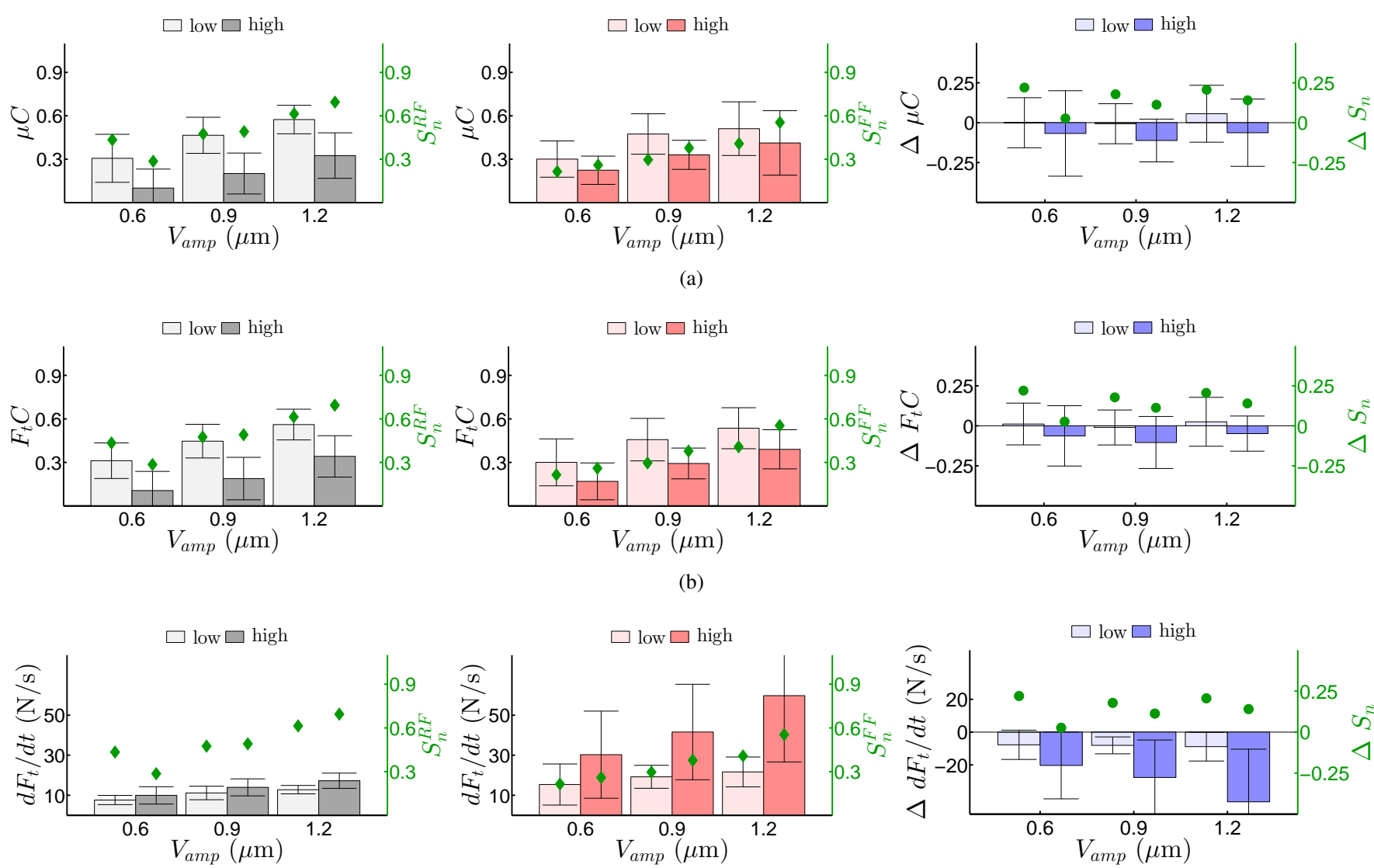

(b)

Fig. 10. Means and standard deviations of various force metrics for $E x p-3$. The first, second, and third column report the results for $R F, F F$, and difference between $R F$ and $F F$, respectively. Green-colored diamonds and circles represent mean values of $S_{n}$ and $\Delta S_{n}$, respectively (Refer to the values on the right axis in each plot).

[44]), which results in higher tangential force and magnitude estimates. Similarly, in $\operatorname{Exp}-3$, despite a decrease in $F_{t} C$, an increase in $d F_{t} / d t$ was a clear indication of increased fingertip stiffness.

In sliding experiments performed above the detection threshold, we observed that $R F$ was more strongly perceived than $F F$, and the difference $\left(\Delta S_{n}=S_{n}^{R F}-S_{n}^{F F}\right)$ was positive. This contrast in tactile perception is perhaps due to the viscoelastic properties of the fingertip and the hysteresis effect. Wang et al. [44] have found that the skin deformed at a high rate when a step force was applied, but restored more slowly when the force was removed. In our experiments, the finger experienced lower tangential force followed by higher one under the condition of $R F$. Consequently, when we rendered $R F$, the finger deformed at a higher rate, which produced a stronger tactile feeling as compared to $F F$. Thus, the results of our experiments and literature suggest that the difference between $R F$ and $F F$ is due to viscoelasticity and not related to slower force transition in $R F$ due to slower discharge of squeeze film [7]. Viscoelastic materials show ratedependent response and relaxation behavior. We argue that the increase in friction was more easily differentiated by subjects during $R F$ due to the rate-dependent behavior compared to the friction drop during $F F$ due to relaxation behavior. If viscoelasticity has an influence on our tactile perception, one anticipates that the experimental results of this study would be affected by finger velocity $\left(f_{v}\right)$, which is not the case. The velocity range covered in our study $(10-70 \mathrm{~mm} / \mathrm{s})$ is relatively small and the effect on tactile perception was not observable. The earlier studies in literature also show that the change in finger friction is observable only when there is a large difference in finger velocities [14]. The experiments performed by Moy et al. [45] indirectly support our claim. They asked the subjects to count the number of ridges scanned under their fingers. When a small ridge was presented before a big one, subjects perceived both ridges successfully. However, in opposite order, the subjects were not able to detect the small ridge. The authors argued that the poor perception of the small ridge after big one was due to the residual deformation of the fingertip due to hysteresis, as observed in viscoelastic materials.

\section{Conclusion And Future Work}

Using an ultrasonic tactile display, we investigated our tactile perception of change in friction. For a sliding-finger, subjects' psychometric curves for rising friction $(R F)$ and falling friction $(F F)$ were almost identical, while they felt the friction change for $F F$ only when they gently pressed their finger on the surface without sliding, as in key press. On 
the other hand, when the sliding experiment was repeated at stimuli levels significantly higher than the detection threshold level, $R F$ was perceived more strongly than $F F$. These results suggest that the factors affecting our tactile perception of friction change for sliding are different than those of nosliding. For sliding-finger, our results suggest that the perceived intensity of friction change was best correlated with tangential force contrast $\left(F_{t} C\right)$ and rate of change in tangential force $\left(d F_{t} / d t\right)$. A simple linear model using only those two metrics estimated the subjects' perception quite well. However, the difference between the correlation coefficients for $F_{t} C$ and $\mu C$ was not significant, as tabulated in table V. Either can be used with $d F_{t} / d t$ to estimate the subjects' perception.

Intensity scaling experiments showed that the finger velocity has little or almost no effect on our tactile perception of change in friction. However, subjects' perception was affected by the transition time (i.e. slope of the friction change) and the normal force. As the transition time was longer (and hence the slope was lower), the strength of their perception was reduced. As the normal force was increased, subjects perceived a stronger change in friction in case of $F F$, though the perceptual difference between $R F$ and $F F$ was reduced. However, the effects of finger velocity, transition time and normal force on threshold levels cannot be ascertained by our experiments. We should also point out that the results of the study should be interpreted carefully due to several factors that may potentially affect the outcomes of the experiments, such as cleaning of finger with alcohol, which reduces the influence of hydrolipid film, finger humidity, a smaller number of subjects, and unbalance between male and female subjects.

We proposed that the perceptual difference between $R F$ and $F F$ during finger sliding was due to viscoelastic properties of fingertip. We supported this claim by relating our results with those of the earlier studies in the literature. In order to provide a further support, skin deformations in fingertip can be analyzed during friction change, using a high-speed camera and computer vision techniques, as done in [6]. Additionally, evaluating the effect of $F_{t} C$ and $d F_{t} / d t$ on our tactile perception of textures and geometrical shapes is another avenue that can be explored in the future.

\section{REFERENCES}

[1] O. Bau, I. Poupyrev, A. Israr, and C. Harrison, "Teslatouch: Electrovibration for touch surfaces," in Proceedings of the 23Nd Annual ACM Symposium on User Interface Software and Technology, UIST '10, (New York, NY, USA), pp. 283-292, ACM, 2010.

[2] T. Watanabe and S. Fukui, "A method for controlling tactile sensation of surface roughness using ultrasonic vibration," in Proceedings of 1995 IEEE International Conference on Robotics and Automation, vol. 1, pp. 1134-1139 vol.1, May 1995.

[3] X. Dai, J. E. Colgate, and M. A. Peshkin, "Lateralpad: A surface-haptic device that produces lateral forces on a bare finger," in 2012 IEEE Haptics Symposium (HAPTICS), pp. 7-14, March 2012.

[4] E. Vezzoli, Z. Vidrih, V. Giamundo, B. Lemaire-Semail, F. Giraud, T. Rodic, D. Peric, and M. Adams, "Friction reduction through ultrasonic vibration part 1: Modelling intermittent contact," IEEE Transactions on Haptics, vol. 10, pp. 196-207, April 2017.

[5] T. Sednaoui, E. Vezzoli, B. Dzidek, B. Lemaire-Semail, C. Chappaz, and M. Adams, "Friction reduction through ultrasonic vibration part 2: Experimental evaluation of intermittent contact and squeeze film levitation," IEEE Transactions on Haptics, vol. 10, pp. 208-216, April 2017.
[6] M. Wiertlewski, R. Fenton Friesen, and J. E. Colgate, "Partial squeeze film levitation modulates fingertip friction," Proceedings of the National Academy of Sciences, vol. 113, no. 33, pp. 9210-9215, 2016.

[7] D. J. Meyer, M. Wiertlewski, M. A. Peshkin, and J. E. Colgate, "Dynamics of ultrasonic and electrostatic friction modulation for rendering texture on haptic surfaces," in 2014 IEEE Haptics Symposium (HAPTICS), pp. 63-67, Feb 2014.

[8] F. Giraud, M. Amberg, and B. Lemaire-Semail, "Merging two tactile stimulation principles: electrovibration and squeeze film effect," in 2013 World Haptics Conference (WHC), pp. 199-203, April 2013.

[9] Y. Rekik, E. Vezzoli, L. Grisoni, and F. Giraud, "Localized haptic texture: A rendering technique based on taxels for high density tactile feedback," in Proceedings of the 2017 CHI Conference on Human Factors in Computing Systems, CHI '17, (New York, NY, USA), pp. 5006-5015, ACM, 2017.

[10] M. J. Adams, B. J. Briscoe, and S. A. Johnson, "Friction and lubrication of human skin," Tribology Letters, vol. 26, pp. 239-253, Jun 2007.

[11] T. André, P. Lefvre, and J.-L. Thonnard, "A continuous measure of fingertip friction during precision grip," Journal of Neuroscience Methods, vol. 179 , no. 2 , pp. $224-229,2009$.

[12] S. Derler, L.-C. Gerhardt, A. Lenz, E. Bertaux, and M. Hadad, "Friction of human skin against smooth and rough glass as a function of the contact pressure," Tribology International, vol. 42, no. 11, pp. 1565 1574, 2009.

[13] S. M. Pasumarty, S. A. Johnson, S. A. Watson, and M. J. Adams, "Friction of the human finger pad: Influence of moisture, occlusion and velocity," Tribology Letters, vol. 44, p. 117, Aug 2011.

[14] M. J. Adams, S. A. Johnson, P. Lefèvre, V. Lévesque, V. Hayward, T. André, and J.-L. Thonnard, "Finger pad friction and its role in grip and touch," Journal of The Royal Society Interface, vol. 10, no. 80 2013.

[15] B. Delhaye, P. Lefèvre, and J.-L. Thonnard, "Dynamics of fingertip contact during the onset of tangential slip," Journal of The Royal Society Interface, vol. 11, no. 100, 2014.

[16] B. Delhaye, A. Barrea, B. B. Edin, P. Lefèvre, and J.-L. Thonnard, "Surface strain measurements of fingertip skin under shearing," Journal of The Royal Society Interface, vol. 13, no. 115, 2016.

[17] T. Sednaoui, E. Vezzoli, B. Dzidek, B. Lemaire-Semail, C. Chappaz, and M. Adams, "Experimental evaluation of friction reduction in ultrasonic devices," in 2015 IEEE World Haptics Conference (WHC), pp. 37-42, June 2015.

[18] A. M. Smith and S. H. Scott, "Subjective scaling of smooth surface friction," Journal of Neurophysiology, vol. 75, no. 5, pp. 1957-1962, 1996.

[19] D. Gueorguiev, S. Bochereau, A. Mouraux, V. Hayward, and J.-L. Thonnard, "Touch uses frictional cues to discriminate flat materials," Scientific Reports, vol. 6, 2016.

[20] E. Samur, J. E. Colgate, and M. A. Peshkin, "Psychophysical evaluation of a variable friction tactile interface," in International Conference on Perception and Cognition in Electronics Media, San Jose, California, 2009.

[21] P.-H. Cornuault, L. Carpentier, M.-A. Bueno, J.-M. Cote, and G. Monteil, "Influence of physico-chemical, mechanical and morphological fingerpad properties on the frictional distinction of sticky/slippery surfaces," Journal of The Royal Society Interface, vol. 12, no. 110, 2015.

[22] W. B. Messaoud, M.-A. Bueno, and B. Lemaire-Semail, "Relation between human perceived friction and finger friction characteristics," Tribology International, vol. 98, pp. 261 - 269, 2016.

[23] M. K. Saleem, C. Yilmaz, and C. Basdogan, "Tactile perception of change in friction on an ultrasonically actuated glass surface," in 2017 IEEE World Haptics Conference (WHC), pp. 495-500, June 2017.

[24] Y. Vardar, A. Isleyen, M. K. Saleem, and C. Basdogan, "Roughness perception of virtual textures displayed by electrovibration on touch screens," in 2017 IEEE World Haptics Conference (WHC), pp. 263268, June 2017

[25] D. Gueorguiev, E. Vezzoli, T. Sednaoui, L. Grisoni, and B. LemaireSemail, "Feeling multiple edges: The tactile perception of short ultrasonic square reductions of the finger-surface friction," in 2017 IEEE World Haptics Conference (WHC), pp. 125-129, June 2017.

[26] J. Monnoyer, E. Diaz, C. Bourdin, and M. Wiertlewski, "Optimal skin impedance promotes perception of ultrasonic switches," in 2017 IEEE World Haptics Conference (WHC), pp. 130-135, June 2017.

[27] A. M. Smith, C. E. Chapman, M. Deslandes, J.-S. Langlais, and M.P. Thibodeau, "Role of friction and tangential force variation in the subjective scaling of tactile roughness," Experimental Brain Research, vol. 144, no. 2, pp. 211-223, 2002. 
[28] X. Chen, F. Shao, C. Barnes, T. Childs, and B. Henson, "Exploring relationships between touch perception and surface physical properties," International Journal of Design, vol. 3, no. 2, pp. 67-76, 2009.

[29] D. Gueorguiev, E. Vezzoli, A. Mouraux, B. Lemaire-Semail, and J.L. Thonnard, "The tactile perception of transient changes in friction," Journal of The Royal Society Interface, vol. 14, no. 137, 2017.

[30] K. Tashiro, Y. Shiokawa, T. Aono, and T. Maeno, "Realization of button click feeling by use of ultrasonic vibration and force feedback," in World Haptics 2009 - Third Joint EuroHaptics conference and Symposium on Haptic Interfaces for Virtual Environment and Teleoperator Systems, pp. 1-6, March 2009.

[31] J. Monnoyer, E. Diaz, C. Bourdin, and M. Wiertlewski, Ultrasonic Friction Modulation While Pressing Induces a Tactile Feedback, pp. 171179. Cham: Springer International Publishing, 2016.

[32] F. Giraud, M. Amberg, and B. Lemaire-Semail, "Design and control of a haptic knob," Sensors and Actuators A: Physical, vol. 196, pp. 78 85, 2013.

[33] W. B. Messaoud, F. Giraud, B. Lemaire-Semail, M. Amberg, and M. A. Bueno, "Amplitude control of an ultrasonic vibration for a tactile stimulator," IEEE/ASME Transactions on Mechatronics, vol. 21, pp. 1692-1701, June 2016.

[34] L. A. Jones and H. Z. Tan, "Application of psychophysical techniques to haptic research," IEEE Transactions on Haptics, vol. 6, pp. 268-284, July 2013.

[35] A. Ramalho, P. Szekeres, and E. Fernandes, "Friction and tactile perception of textile fabrics," Tribology International, vol. 63, pp. 29 $-33,2013$.

[36] Y. Tanaka, W. M. Bergmann Tiest, A. M. L. Kappers, and A. Sano, "Contact force and scanning velocity during active roughness perception," PLOS ONE, vol. 9, pp. 1-11, 032014.

[37] R. Fagiani, F. Massi, E. Chatelet, J. P. Costes, and Y. Berthier, "Contact of a finger on rigid surfaces and textiles: Friction coefficient and induced vibrations," Tribology Letters, vol. 48, pp. 145-158, Nov 2012.

[38] D. Gueorguiev, " Tactile perception of natural and simulated textures: a psychophysical approach. $\mathrm{PhD}$ thesis, Université catholique de Louvain, 2016.

[39] Y. Vardar, B. Guclu, and C. Basdogan, "Effect of waveform on tactile perception by electrovibration displayed on touch screens," IEEE Transactions on Haptics, vol. 10, no. 4, pp. 488-499, 2017.

[40] A. Guzererler, W. R. Provancher, and C. Basdogan, "Perception of skin stretch applied to palm: Effects of speed and displacement," in Haptics: Perception, Devices, Control, and Applications: 10th International Conference, EuroHaptics 2016, London, UK, July 4-7, 2016, Proceedings, Part I, pp. 180-189, Cham: Springer International Publishing, 2016.

[41] P. Shull, K. Bark, and M. Cutkosky, "Skin nonlinearities and their effect on user perception for rotational skin stretch," in 2010 IEEE Haptics Symposium, pp. 77-82, March 2010.

[42] M. Paré, H. Carnahan, and A. M. Smith, "Magnitude estimation of tangential force applied to the fingerpad," Experimental Brain Research, vol. 142, pp. 342-348, Feb 2002.

[43] V. A. Ho and S. Hirai, "From localized shearing to localized slippage perception," in 2016 International Symposium on Experimental Robotics, pp. 443-453, Cham: Springer International Publishing, 2017.

[44] Q. Wang and V. Hayward, "In vivo biomechanics of the fingerpad skin under local tangential traction," Journal of Biomechanics, vol. 40, no. 4, pp. $851-860,2007$.

[45] G. Moy, U. Singh, E. Tan, and R. S. Fearing, "Human psychophysics for teletaction system design," Haptics-e Journal, vol. 1, no. 3, 2000.

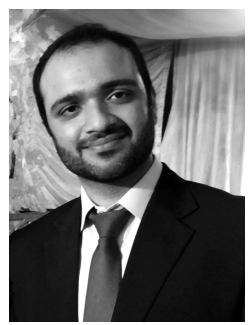

Muhammad Khurram Saleem received his BS and MS in Mechatronics Engineering from University of Engineering and Technology, Lahore, Pakistan. $\mathrm{He}$ is currently working toward a Ph.D. degree in the Department of Mechanical Engineering with the Robotics and Mechatronics Lab, Koc University, Turkey. His research interests include haptics and mechatronic systems.

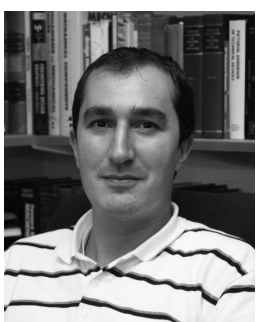

Cetin Yilmaz received his BS degree with high honors in Mechanical Engineering from Bogazici University in 2000 . He pursued his graduate study at University of Michigan between 2000 and 2005. In 2001 and 2002 he completed his MS degrees in Mechanical Engineering and Mathematics, respectively. In 2005 he received his $\mathrm{PhD}$ degree in Mechanical Engineering. Between 2005 and 2007 he worked as a post-doctoral researcher at University of Michigan. Currently, he is an Associate Professor of Mechanical Engineering at Bogazici University. His research interests include multi-body dynamics, acoustics and vibrations, phononic band gap structures, and design

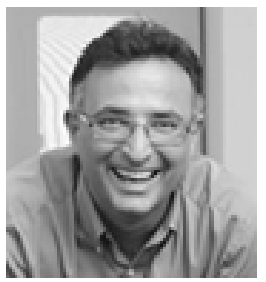

Cagatay Basdogan received the Ph.D. degree in mechanical engineering from Southern Methodist University in 1994. He is a faculty member in the mechanical engineering and computational sciences and engineering programs of Koc University, Istanbul, Turkey. He is also the director of the Robotics and Mechatronics Laboratory at Koc University. Before joining Koc University, he worked at NASAJPL/Caltech, MIT, and Northwestern University Research Park. His research interests include haptic interfaces, robotics, mechatronics, biomechanics, medical simulation, computer graphics, and multi-modal virtual environments. $\mathrm{He}$ is currently the associate editor in chief of IEEE Transactions on Haptics and serves in the editorial boards of IEEE Transactions on Mechatronics, Presence: Teleoperators and Virtual Environments, and Computer Animation and VirtualWorlds journals. 